\title{
DIVERGENCE $L^{2}$-COERCIVITY INEQUALITIES.
}

\author{
GILES AUCHMUTY
}

\begin{abstract}
This paper describes sharp $L^{2}$-coercivity inequalities for the divergence operator on bounded Lipschitz regions in $\mathbb{R}^{n}$. The inequalities are given in a manner which scales under dilations of the region. The optimal constants in the inequality are identified as the least eigenvalue of a biharmonic problem or else of a non-standard problem for the Dirichlet Laplacian. When $n=2$, a coercivity result for the curl operator is also obtained.
\end{abstract}

\section{INTRODUCTION}

An important class of estimates for the analysis of elliptic boundary value problems are Friedrichs' type inequalities such as

$$
\int_{\Omega}|\nabla \varphi|^{2} d^{n} x+\int_{\partial \Omega}|\varphi|^{2} d \sigma \geq C_{0} \int_{\Omega}|\varphi|^{2} d^{n} x \quad \text { for all } \varphi \in H^{1}(\Omega) .
$$

Here $\Omega$ is a bounded region with a sufficiently nice boundary. See Necas [9] theorem 1.9 and Hellwig [7], Section 5.3 for proofs of this result and references to the original literature. For a recent discussion of generalizations, including $L^{p}$ versions, of this inequality see Auchmuty [3].

For the analysis of boundary value problems involving vector fields, it is natural to ask about possible analogues of this inequality when $\varphi$ is replaced by a vector field $v$ and the gradient operator is replaced by $\operatorname{div} v$ or $\operatorname{curl} v$. Some such inequalities are described in Monk [8] chapter 3 for use in the analysis of Maxwell's equations. In this paper sharp inequalities of the form

$$
\int_{\Omega}|\operatorname{div} v|^{2} d^{n} x+|\Omega|^{-1}\left(\int_{\partial \Omega} v \cdot \nu d \sigma\right)^{2} \geq C_{2} \int_{\Omega} \rho|v|^{2} d^{n} x
$$

will be proved. Here $v$ lies in a specific subspace of $H(\operatorname{div}, \Omega)$ and the optimal constant $\mathrm{C}_{2}$ will be characterized. The factor in front of the boundary integral here is chosen so that this inequality scales under dilations as described in section 3. This inequality is sharper than those described in [8] as it does not require that the boundary flux $v \cdot \nu$ be in $L^{2}(\partial \Omega, d \sigma)$.

In section 4 , a representation theorem is described which enables the characterization of the subspace of fields for which this inequality holds. Then the existence of a finite constant $C_{2}$ such that (1.2) holds is proved using variational arguments in section

\footnotetext{
Date: January 2, 2005.
} 
5. The following section 6 describes an unconstrained variational principle for the optimal constant $C_{2}$ which is then used to show that the constant is the least eigenvalue of a biharmonic boundary value problem.

When $\rho(x) \equiv 1$, this eigenproblem can be reduced to a non-standard eigenproblem for the Dirichlet Laplacian on $\Omega$. As a consequence, upper and lower bounds on $C_{2}$ are found in terms of the first eigenvalue of the Dirichlet eigenproblem on $\Omega$.

In section 8 a similar inequality for the curl operator with the same optimal constant is proved when $n=2$. Namely

$$
\int_{\Omega}|\operatorname{curl} v|^{2} d^{2} x+|\Omega|^{-1}\left(\int_{\partial \Omega}(v \cdot \tau) d s\right)^{2} \geq C_{2} \int_{\Omega} \rho|v|^{2} d^{2} x .
$$

This holds for all fields in a subspace of $H(\operatorname{curl}, \Omega)$. Here $\tau$ is a unit tangent field on the boundary $\partial \Omega$ while $d s$ represents arc length.

In this paper, we will not try to describe the most general inequalities of this type involving the divergence operator. We shall, however, identify the optimal constants in these inequalities and the associated extremal fields.

\section{Definitions and Assumptions.}

Let $\Omega$ be a non-empty, bounded, connected, open subset of $\mathbb{R}^{n}, n \geq 2$, with boundary $\partial \Omega$. Such a set $\Omega$ is called a region. Its closure is denoted $\bar{\Omega}$ and its boundary is $\partial \Omega:=\bar{\Omega} \backslash \Omega$. Points in $\Omega$ will be described using Cartesian coordinates $x:=\left(x_{1}, x_{2}, \ldots, x_{n}\right)$. When $x, y \in \mathbb{R}^{n}$, their scalar product and Euclidean norm are denoted $x \cdot y,|x|$ respectively.

Let $L^{2}(\Omega)$ be the usual real Lebesgue space of all functions $\varphi: \Omega \rightarrow[-\infty, \infty]$ which are square integrable with respect to Lebesgue measure on $\Omega$. Similarly $L^{2}\left(\Omega ; \mathbb{R}^{n}\right)$ is the class of all vector fields $v$ on $\Omega$ whose components $v_{j}$ all are in $L^{2}(\Omega)$. These are real Hilbert spaces under the usual inner products

$$
\langle\varphi, \psi\rangle:=\int_{\Omega} \varphi \psi d^{n} x \text { and }\langle u, v\rangle:=\int_{\Omega} u \cdot v d^{n} x
$$

The weak j-th derivative of a function $\varphi$ is denoted $D_{j} \varphi$ and its gradient is the vector field $\nabla \varphi:=\left(D_{1} \varphi, D_{2} \varphi, \ldots, D_{n} \varphi\right)$. The Sobolev space $H^{1}(\Omega)$ is defined to be the space of all functions in $L^{2}(\Omega)$, whose gradient is also in $L^{2}\left(\Omega ; \mathbb{R}^{n}\right)$. This is a real Hilbert space under the $H^{1}-$ inner product

$$
[\phi, \psi]_{1}:=\int_{\Omega}[\phi(x) \psi(x)+\nabla \varphi(x) \cdot \nabla \psi(x)] d^{n} x .
$$

The space $H_{0}^{1}(\Omega)$ is the closure of $C_{c}^{\infty}(\Omega)$ with respect to the norm $(2.2)$. When $\Omega$ is bounded an equivalent inner product on $H_{0}^{1}(\Omega)$ will be

$$
\langle\varphi, \psi\rangle_{1}:=\int_{\Omega} \nabla \varphi \cdot \nabla \psi d^{n} x
$$


The divergence of a vector field $v \in L^{2}\left(\Omega ; \mathbb{R}^{n}\right)$ is the distribution $T_{v}$ defined by

$$
T_{v}(\varphi):=-\int_{\Omega} v \cdot \nabla \varphi d^{n} x \quad \text { for all } \varphi \in C_{c}^{\infty}(\Omega) .
$$

Suppose there is a function $\rho \in L_{l o c}^{1}(\Omega)$ such that

$$
T_{v}(\varphi)=-\int_{\Omega} \rho \varphi d^{n} x \quad \text { for all } \varphi \in C_{c}^{\infty}(\Omega) .
$$

Then we say that $\rho=\operatorname{div} v$ on $\Omega$.

Define $H(\operatorname{div}, \Omega)$ to be the subspace of all fields in $L^{2}\left(\Omega ; \mathbb{R}^{n}\right)$ having $\operatorname{div} v$ in $L^{2}(\Omega)$. $H(\operatorname{div}, \Omega)$ is a real Hilbert space under the inner product

$$
[v, w]_{\operatorname{div}}:=\int_{\Omega}[v(x) \cdot w(x)+\operatorname{div} v(x) \operatorname{div} w(x)] d^{n} x .
$$

The associated norm will be denoted $\|\cdot\|_{\text {div }}$.

The field $v \in L^{2}(\Omega)$ is said to be solenoidal, or divergence-free, whenever

$$
\int_{\Omega} v \cdot \nabla \varphi d^{n} x=0 \quad \text { for all } \varphi \in C_{c}^{\infty}(\Omega) .
$$

That is the (weak) divergence of $v$ is zero on $\Omega$. The class of all solenoidal vector fields in $H(\operatorname{div}, \Omega)$ will be denoted $N(\operatorname{div})$ and is a closed subspace of $L^{2}\left(\Omega ; \mathbb{R}^{n}\right)$ and of $H(\operatorname{div}, \Omega)$.

For our analysis we also require some mild regularity conditions on $\Omega$ and $\partial \Omega$. Our basic criteria is that the boundary $\partial \Omega$ is Lipschitz in the sense of Evans and Gariepy, [5], section 4.2 .

Let $\sigma, d \sigma$ represent Hausdorff $(n-1)$-dimensional measure and integration with respect to this measure respectively. When $\partial \Omega$ is Lipschitz, the unit outer normal $\nu(x)$ is defined $\sigma$ a.e. on $\partial \Omega$ and the boundary traces of functions in $W^{1, p}(\Omega)$ are well-defined. See section 4.3 loc. cit. In particular, the Gauss-Green theorem holds in the form

$$
\int_{\Omega} D_{j} \psi d^{n} x=\int_{\partial \Omega} \psi \nu_{j} d \sigma \quad \text { for all } 1 \leq j \leq n, \psi \in W^{1,1}(\Omega) .
$$

This follows from theorem 1 , section 4.3 of [5] with $\mathrm{p}=1$. We will generally require that $\Omega$ satisfy

Condition A1: $\Omega$ is a bounded open region and $\partial \Omega$ is a finite union of Lipschitz surfaces, each with finite surface area.

When $v, \operatorname{div} v$ are in $L^{1}\left(\Omega ; \mathbb{R}^{n}\right), L^{1}(\Omega)$ respectively this implies that the divergence theorem holds on the region $\Omega$. Namely

$$
\int_{\Omega} \operatorname{div} v d^{n} x=\int_{\partial \Omega} v \cdot \nu d \sigma .
$$

This follows from (2.8) by substituting $v_{j}$ for $\psi$ and summing over $j$.

The function $\rho$ will be required to satisfy 
Condition A2: $\rho \in L^{\infty}(\Omega)$ satisfies $\rho(x) \geq 0$ a.e. on $\Omega$ and $\int_{\Omega} \rho d x>0$.

\section{SCALing Under Dilations}

There are a variety of forms for the inequality (1.2). Consider the functionals $\mathcal{F}, \mathcal{P}: H(\operatorname{div}, \Omega) \rightarrow[0, \infty)$ defined by

$$
\begin{aligned}
\mathcal{F}(v) & :=\int_{\Omega}|\operatorname{div} v|^{2} d^{n} x+|\Omega|^{-1}\left(\int_{\Omega} \operatorname{div} v d^{n} x\right)^{2} \quad \text { and } \\
\mathcal{P}(v) & :=\int_{\Omega} \rho(x)|v(x)|^{2} d^{n} x .
\end{aligned}
$$

We will describe a subspace $\mathrm{X}$ of $H(\operatorname{div}, \Omega)$ and a constant $C_{2}>0$ such that

$$
\mathcal{F}(v) \geq C_{2} \mathcal{P}(v) \quad \text { for all } v \in X .
$$

Note that the divergence theorem (2.9) implies that this $\mathcal{F}(v)$ is equal to the left hand side of (1.2). From Schwarz inequality

$$
\left(\int_{\partial \Omega} v \cdot \nu d \sigma\right)^{2} \leq|\partial \Omega| \int_{\partial \Omega}|v \cdot \nu|^{2} d \sigma
$$

When $v \in H(\operatorname{div}, \Omega)$ and $|\Omega|$ is finite, the left hand side of this inequality is always finite - but the right hand side need not be. Thus (3.3) is significantly better than the (3d) result described in corollary 3.51 of [8].

The coefficient in the definition of $\mathcal{F}$ was chosen so that the inequality scales under dilations. Given a reference region $\Omega$, and $L>0$, define $\Omega_{L}:=\{L x: x \in \Omega\}$. Consider the dilation operator $\mathcal{S}_{L}: H(\operatorname{div}, \Omega) \rightarrow H\left(\operatorname{div}, \Omega_{L}\right)$ defined by

$$
\mathcal{S}_{L} v(y):=v_{L}(y):=v(y / L) \quad \text { for } \quad y \in \Omega_{L} .
$$

This is a linear isomorphism and we have

$$
\operatorname{div}_{y} v_{L}(y)=L^{-1} \operatorname{div}_{x} v(y / L) \quad \text { for each } v \in H(\operatorname{div}, \Omega), L>0 .
$$

For $L>0$, define the functional $\mathcal{P}_{L}: L^{2}\left(\Omega_{L} ; \mathbb{R}^{n}\right) \rightarrow[0, \infty]$ by

$$
\mathcal{P}_{L}\left(v_{L}\right):=\int_{\Omega_{L}} \rho_{L}(y)\left|v_{L}(y)\right|^{p} d y
$$

with $\rho_{L}:=\mathcal{S}_{L} \rho$. This functional satisfies

$$
\mathcal{P}_{L}\left(v_{L}\right)=L^{n} \mathcal{P}(v) \quad \text { for each } \quad v \in L^{2}\left(\Omega ; \mathbb{R}^{n}\right) .
$$

The change of variables rule and (3.6) yield

$$
\begin{gathered}
\int_{\Omega_{L}}\left|\operatorname{div} v_{L}(y)\right|^{2} d^{n} y=L^{n-2} \int_{\Omega}|\operatorname{div} v(x)|^{2} d^{n} x . \\
\int_{\Omega_{L}} \operatorname{div} v_{L}(y) d^{n} y=L^{n-1} \int_{\Omega} \operatorname{div} v(x) d^{n} x .
\end{gathered}
$$


If there is a constant $C_{2}$ such that (3.3) holds on $\Omega$, then (3.8)-(3.10) imply

$$
\int_{\Omega_{L}}\left|\operatorname{div} v_{L}\right|^{2} d^{n} y+\left|\Omega_{L}\right|^{-1}\left|\int_{\Omega_{L}} \operatorname{div} v_{L} d^{n} y\right|^{2} \geq C_{2} L^{-2} \int_{\Omega_{L}} \rho_{L}(y)\left|v_{L}\right|^{2} d^{n} y
$$

Thus this particular combination of terms provides a simple scaling law under dilations of the region.

\section{Potentials and Allowable Fields}

Clearly the inequality (3.3) cannot hold when $v$ is solenoidal as $\mathcal{F}(v)=0$ for such fields. We shall show, however, that the inequality holds when $\mathrm{X}$ is taken to be the orthogonal complement of $N(\operatorname{div})$ in $H(\operatorname{div}, \Omega)$. First note that $N(\operatorname{div})$ is a different space that the usual space of divergence-free fields studied in texts on the Navier-Stokes equations. For example, theorem 1.1 in chapter 3 of Galdi [6], describes the complement of the space of fields in the closure of the smooth solenoidal fields with compact support on $\Omega$. This is a proper subspace of $N(\operatorname{div})$, so its $L^{2}$ - orthogonal complement is a different (and larger) subspace of $H(\operatorname{div}, \Omega)$.

The Laplacian is the linear differential operator defined by $\Delta \varphi:=\operatorname{div}(\nabla \varphi)$. Define $H_{0}(\Delta, \Omega)$ to be the subspace of all functions $\varphi$ in $H_{0}^{1}(\Omega)$ having $\Delta \varphi$ in $L^{2}(\Omega)$. This is a real Hilbert space under the inner product

$$
[\varphi, \psi]_{\Delta}:=\int_{\Omega} \Delta \varphi \Delta \psi d^{n} x
$$

The corresponding norm is denoted $\|\cdot\|_{\Delta}$.

Define $G_{0}^{1}(\Omega):=\left\{\nabla \varphi: \varphi \in H_{0}(\Delta, \Omega)\right\}$, then $G_{0}^{1}(\Omega)$ is a subspace of $H(\operatorname{div}, \Omega)$. The important property for our purposes is the following.

Theorem 4.1. Assume (A1) holds, then $G_{0}^{1}(\Omega)$ is the orthogonal complement of $N(\operatorname{div})$ in $H(\operatorname{div}, \Omega)$

Proof. When $v \in H(\operatorname{div}, \Omega)$ and $\varphi \in H_{0}^{1}(\Omega)$, apply the divergence theorem (2.9) to $\varphi v$ to see that

$$
\int_{\Omega} v \cdot \nabla \varphi d^{n} x+\int_{\Omega} \varphi \operatorname{div} v d^{n} x=0
$$

Thus the subspaces $G_{0}^{1}(\Omega)$ and $N(\operatorname{div})$ are $L^{2}$-orthogonal and orthogonal in $H(\operatorname{div}, \Omega)$.

This result will be proved by establishing a representation theorem for fields in $H(\operatorname{div}, \Omega)$ using saddle point methods. First note that div is a continuous linear mapping of $H(\operatorname{div}, \Omega)$ into $L^{2}(\Omega)$, so $N(\operatorname{div})$ will be a closed subspace of $H(\operatorname{div}, \Omega)$.

Given $v \in H(\operatorname{div}, \Omega)$, consider the problem of finding a saddle point of the functional $\mathcal{L}: H(\operatorname{div}, \Omega) \times H_{0}(\Delta, \Omega) \rightarrow \mathbb{R}$ defined by

$$
\mathcal{L}(u, \varphi):=\int_{\Omega}\left[|u-v|^{2}-2 \varphi \operatorname{div} u\right] d^{n} x .
$$


Maximizing this on $H_{0}^{1}(\Omega)$, one observes that

$$
\sup _{\varphi \in H_{0}^{1}(\Omega)} \mathcal{L}(u, \varphi)=\|u-v\|^{2} \quad \text { if } u \in N(\operatorname{div}) .
$$

When $u \notin N($ div $)$, this supremum is $\infty$.

The primal problem associated with this functional is to minimize $\|u-v\|^{2}$ subject to $u \in N$ (div). This has a unique solution $\hat{u}$ which is the projection of $v$ onto the closed subspace $N(\operatorname{div})$ of $H(\operatorname{div}, \Omega)$.

Now consider the problem of evaluating the dual functional $\mathcal{G}: H_{0}(\Delta, \Omega) \rightarrow \mathbb{R}$ defined by

$$
\mathcal{G}(\varphi):=\inf _{u \in H(\operatorname{div}, \Omega)} \int_{\Omega}\left[|u-v|^{2}-2 \varphi \operatorname{div} u\right] d^{n} x
$$

This is a simple quadratic variational problem and the integral is minimized when $u=v-\nabla \varphi$. Substitute this back in the integrand to find that

$$
-\mathcal{G}(\varphi)=\int_{\Omega}\left[|\nabla \varphi|^{2}+2 \varphi \operatorname{div} v\right] d^{n} x
$$

The dual problem is to maximize $\mathcal{G}$ on $H_{0}(\Delta, \Omega)$. $-\mathcal{G}$ is a convex functional so any critical point of $\mathcal{G}$ is a maximizer of $\mathcal{G}$ on $H_{0}(\Delta, \Omega)$. An elementary calculation shows that these critical points are solutions of

$$
\langle D \mathcal{G}(\varphi), \psi\rangle=\int_{\Omega}(\nabla \varphi \cdot \nabla \psi+\psi \operatorname{div} v) d^{n} x=0 \quad \text { for all } \psi \in H_{0}(\Delta, \Omega) .
$$

This is a well-known problem which has a unique solution $\hat{\varphi}$ in $H_{0}^{1}(\Omega)$, which will be in $H_{0}(\Delta, \Omega)$ when $v \in H(\operatorname{div}, \Omega)$. Note that (4.6) is the weak version of the equation

$$
\Delta \varphi=\operatorname{div} v \quad \text { on } \Omega \text { with } \varphi=0 \text { on } \partial \Omega \text {. }
$$

Putting $\psi=\hat{\varphi}$ in (4.6) and substituting leads to

$$
\mathcal{G}(\hat{\varphi})=\int_{\Omega}|\nabla \hat{\varphi}|^{2} d^{n} x
$$

Define $\hat{u}:=v-\nabla \hat{\varphi}$, then $\hat{u} \in N(\operatorname{div})$ and

$$
\|\hat{u}-v\|^{2}=\|\nabla \hat{\varphi}\|^{2}
$$

This implies that $\hat{u}$ is a solution of the primal problem and that $(\hat{u}, \hat{\varphi})$ is a saddle point of this Lagrangian from theorem 3.1 of [1].

That is, an arbitrary $v=\hat{u}+\nabla \hat{\varphi}$ is the sum of a field in $N(\operatorname{div})$ and one in $G_{0}^{1}(\Omega)$. Since the solution of (4.6) is unique, it follows that this $\hat{\varphi}$, and thus the splitting is unique. Since for each $v \in H(\operatorname{div}, \Omega)$ there is a $\nabla \hat{\varphi} \in G_{0}^{1}(\Omega)$, then from corollary 3.3 of [2], $G_{0}^{1}(\Omega)$ is a closed subspace of $H(\operatorname{div}, \Omega)$ and the proof is complete.

This result may be summarized by the decomposition

$$
H(\operatorname{div}, \Omega)=G_{0}^{1}(\Omega) \oplus N(\operatorname{div}) .
$$


The subspaces on this right hand side are closed and they are both $L^{2}$-orthogonal and orthogonal with respect to the $H(\operatorname{div}, \Omega)$ inner product.

In view of this characterization of $G_{0}^{1}(\Omega)$, one sees that the inequality (3.3) holds for all $v \in G_{0}^{1}(\Omega)$ if and only if there is a constant $C_{2}>0$ such that

$$
\int_{\Omega}|\Delta \varphi|^{2} d^{n} x+|\Omega| M(\Delta \varphi)^{2} \geq C_{2} \int_{\Omega} \rho|\nabla \varphi|^{2} d^{n} x
$$

for all $\varphi \in H_{0}(\Delta, \Omega)$. Here

$$
M(\chi):=|\Omega|^{-1} \int_{\Omega} \chi d^{n} x
$$

is the mean value of the function $\chi$ on $\Omega$. To prove this inequality we will use the following compact imbedding theorem.

Theorem 4.2. Assume (A1) holds, then $H_{0}(\Delta, \Omega)$ is compactly imbedded in $H_{0}^{1}(\Omega)$.

Proof. Let $\left\{e_{k}: k \geq 1\right\}$ be an $L^{2}$ - orthonormal basis of $H_{0}^{1}(\Omega)$ consisting of eigenfunctions of the Dirichlet Laplacian on $\Omega$. Let $\left\{\lambda_{k}: k \geq 1\right\}$ be the corresponding increasing family of eigenvalues. When (A1) holds there is such a basis and $\operatorname{Lim}_{k \rightarrow \infty} \lambda_{k}=\infty$. See [4], section 6.5, theorem 1.

Suppose $\varphi \in H_{0}^{1}(\Omega)$ and $\varphi=\sum_{k=1}^{\infty} c_{k} e_{k}$ with $c_{k}:=\left\langle\varphi, e_{k}\right\rangle$ be the usual $L^{2}-$ eigenfunction expansion. From Parseval's theorem and the expression (2.3) for the inner product on $H_{0}^{1}(\Omega)$, we see that

$$
\|\nabla \varphi\|_{2}^{2}=\sum_{k=1}^{\infty} \lambda_{k} c_{k}^{2} .
$$

Similarly, using (4.1) and some elementary identities, we see that

$$
\|\varphi\|_{\Delta}^{2}=\sum_{k=1}^{\infty} \lambda_{k}\left(1+\lambda_{k}\right) c_{k}^{2}
$$

To show the imbedding is compact, we prove that the unit ball $\mathrm{B}$ in $H_{0}(\Delta, \Omega)$ is precompact in $H_{0}^{1}(\Omega)$ by showing that for any $\epsilon>0$, B has a finite $\epsilon$-net in $H_{0}^{1}(\Omega)$. Choose $\mathrm{K}$ sufficiently large that $\lambda_{K+1}>2 / \epsilon$. Let $E_{K}$ be the subspace spanned by $\left\{e_{1}, \ldots, e_{K}\right\}$. Then $B \cap E_{K}$ is a bounded compact subset of $H_{0}^{1}(\Omega)$. Let $P_{K} \varphi$ be the projection of $\varphi$ onto $E_{K}$. From (4.11) this has $H_{0}^{1}(\Omega)$ norm given by

$$
\left\|\varphi-P_{K} \varphi\right\|^{2}=\sum_{k=K+1}^{\infty} \lambda_{k} c_{k}^{2}
$$

Since the $\lambda_{k}$ are increasing, this left hand side is

$$
\leq \lambda_{K+1}{ }^{-1}\left(\sum_{k=K+1}^{\infty} \lambda_{k}^{2} c_{k}^{2}\right)<\lambda_{K+1}{ }^{-1}<\epsilon / 2 .
$$

for $\varphi \in B$. Hence B has a finite $\epsilon$-net in $H_{0}^{1}(\Omega)$ as claimed. 


\section{Variational Principles for $C_{2}$.}

The optimal constant $C_{2}$ in (4.10) will now be characterized using a variational principle. Let $\mathrm{C}$ be the subset of functions in $H_{0}(\Delta, \Omega)$ which satisfy

$$
\mathcal{F}_{1}(\varphi):=\int_{\Omega}|\Delta \varphi|^{2} d^{n} x+|\Omega| M(\Delta \varphi)^{2} \leq 1
$$

Let $\mathcal{P}_{1}$ be the functional on $H_{0}(\Delta, \Omega)$ defined by

$$
\mathcal{P}_{1}(\varphi):=\int_{\Omega} \rho|\nabla \varphi|^{2} d^{n} x
$$

Consider the variational problem of maximizing $\mathcal{P}_{1}$ on $\mathrm{C}$ and determining

$$
\beta:=\sup _{\varphi \in C} \mathcal{P}_{1}(\varphi)
$$

To describe the existence of solutions of this variational problem, some elementary properties of these functionals are needed.

Proposition 5.1. Assume (A1) holds, then

(i). $\mathcal{F}_{1}$ is continuous, convex and coercive on $H_{0}(\Delta, \Omega)$.

(ii). When (A2) holds, then $\mathcal{P}_{1}$ is convex and weakly continuous on $H_{0}(\Delta, \Omega)$.

(iii). $\quad \mathcal{F}_{1}, \mathcal{P}_{1}$ are $G$-differentiable and their $G$-derivatives are given by

$$
\begin{aligned}
\left\langle D \mathcal{F}_{1}(\varphi), \psi\right\rangle & =2 \int_{\Omega} \Delta \varphi \Delta \psi d^{n} x+2|\Omega| M(\Delta \varphi) M(\Delta \psi) \\
\left\langle D \mathcal{P}_{1}(\varphi), \psi\right\rangle & =2 \int_{\Omega} \rho \nabla \varphi \cdot \nabla \psi d^{n} x .
\end{aligned}
$$

Proof. (i) Apply Schwarz' inequality to $M(\Delta \varphi)$, then

$$
|M(\Delta \varphi)|^{2} \leq|\Omega|^{-1}\|\Delta \varphi\|_{2}^{2} .
$$

Use this and (4.1) in (5.1) then

$$
\|\varphi\|_{\Delta}^{2} \leq \mathcal{F}_{1}(\varphi) \leq 2\|\varphi\|_{\Delta}^{2}
$$

This, and the fact that $\mathcal{F}_{1}$ is quadratic, implies (i).

(ii) $\mathcal{P}_{1}$ is convex as it is quadratic and positive. If $\left\{\varphi_{m}: m \geq 1\right\}$ is a sequence which converges weakly to $\hat{\varphi}$ in $H_{0}(\Delta, \Omega)$ then, from theorem $4.2, \varphi_{m}$ converges strongly to $\hat{\varphi}$ in $H_{0}^{1}(\Omega)$. When (A2) holds, $\mathcal{P}_{1}$ is continuous on $H_{0}^{1}(\Omega)$, so it will be weakly continuous on $H_{0}(\Delta, \Omega)$.

(iii) Evaluate $t^{-1}\left[\mathcal{F}_{1}(\varphi+t \psi)-\mathcal{F}_{1}(\varphi)\right]$ and take the limit as $\mathrm{t}$ goes to zero, to obtain the derivative of $\mathcal{F}_{1}$. Similarly for $\mathcal{P}_{1}$.

Theorem 5.2. Assume (A1) and (A2) hold, then $\beta$ is strictly positive and finite and there are non-zero maximizers $\pm \varphi_{1}$ of $\mathcal{P}_{1}$ on $C$. 
Proof. The set $\mathrm{C}$ is closed and bounded since $\mathcal{F}_{1}$ is continuous and coercive. It is convex as $\mathcal{F}_{1}$ is convex, thus $\mathrm{C}$ will be weakly compact in the Hilbert space $H_{0}(\Delta, \Omega)$. Since $\mathcal{P}_{1}$ is weakly continuous, it will attain its supremum on $\mathrm{C}$ and the value $\beta$ is finite. If $\beta=0$, then every function in $\mathrm{C}$ must have $\nabla \varphi=0$ on the support of $\rho$. This is impossible when (A2) holds.

Since both $\mathcal{F}_{1}, \mathcal{P}_{1}$ are even, if $\varphi_{1}$ is a maximizer, so is $-\varphi_{1}$. The maximizers also must have $\mathcal{F}_{1}\left(\varphi_{1}\right)=1$ by homogeniety.

This theorem enables the proof of the validity of the coercivity inequality (4.10) and the identification of the optimal value of $C_{2}$.

Corollary 5.3. When (A1), (A2) hold, then (4.10) holds for all $\varphi \in H_{0}(\Delta, \Omega)$ with $C_{2}=\beta^{-1}$. In this case, equality holds in (4.10) when $\varphi$ is a multiple of a maximizer of $\mathcal{P}_{1}$ on $C$.

Proof. When $\varphi \neq 0$ is in $H_{0}(\Delta, \Omega)$, then $c \varphi$ will be in $\mathrm{C}$ provided $|c| \leq \mathcal{F}_{1}(\varphi)^{-1 / 2}$. Thus $\mathcal{P}_{1}(c \varphi) \leq \beta$ for such c. Multiply both sides by $\mathcal{F}_{1}(\varphi)$, to find that

$$
\mathcal{P}_{1}(\varphi) \leq \beta \mathcal{F}_{1}(\varphi) \quad \text { for all } \varphi \in H_{0}(\Delta, \Omega)
$$

so (4.10) will hold with $C_{2}:=\beta^{-1}$. The last sentence follows directly.

These results may be restated in terms of the divergence coercivity inequality of section 1.

Theorem 5.4. Assume (A1) and (A2) hold, then (1.2) holds for all $v \in H(\operatorname{div}, \Omega)$ which are $L^{2}$ - orthogonal to $N(\operatorname{div})$ and with $C_{2}=\beta$ as above.

Proof. The previous corollary shows that (4.10) holds with this value of $C_{2}$. That is (1.2) holds for all $v \in G_{0}^{1}(\Omega)$ and moreover equality will hold here when $v= \pm \nabla \varphi_{1}$. This theorem then follows from theorem 4.1.

\section{The Optimal constant as an Eigenvalue}

We have just seen that the optimal constant in (1.2) may be characterized in terms of the value of an inequality constrained variational principle. Here we shall show that it is the least eigenvalue of an unusual eigenproblem for the biharmonic operator.

This eigenproblem arises from the extremality conditions satisfied by the maximizers of $\mathcal{P}_{1}$ on $\mathrm{C}$. Instead of using a theory based on multipliers, the following analysis uses a different variational principle to obtain the equations satisfied by the maximizers of $\mathcal{P}_{1}$ on $\mathrm{C}$.

Consider the functional $\mathcal{J}: H_{0}(\Delta, \Omega) \rightarrow \mathbb{R}$ defined by

$$
\mathcal{J}(\varphi):=\mathcal{F}_{1}(\varphi)^{2}-2 \mathcal{P}_{1}(\varphi)
$$

and the unconstrained problem of finding the infimum of $\mathcal{J}$ on $H_{0}(\Delta, \Omega)$. The basic results about this problem may be summarized as follows. 
Theorem 6.1. Assume A1, A2 hold with $\beta, \mathcal{J}$ defined by (5.3) and (6.1). Then $\mathcal{J}$ is weakly l.s.c. and coercive on $H_{0}(\Delta, \Omega)$ and attains its infimum at points $\pm \tilde{\varphi}$ in $H_{0}(\Delta, \Omega)$ where $\tilde{\varphi}=\beta^{1 / 2} \hat{\varphi}$ and $\hat{\varphi}$ is a maximizer of $\mathcal{P}_{1}$ on $C$. Moreover $\mathcal{F}_{1}(\tilde{\varphi})=$ $\beta, \mathcal{P}_{1}(\tilde{\varphi})=\beta^{2}$ and $\mathcal{J}(\tilde{\varphi})=-\beta^{2}$.

Proof. This is a special case of theorem 5.3 of [3].

When $\mathcal{F}_{1}, \mathcal{P}_{1}$ are G-differentiable on $H_{0}(\Delta, \Omega)$, so is $\mathcal{J}$ with

$$
D \mathcal{J}(\varphi)=2\left[\mathcal{F}_{1}(\varphi) D \mathcal{F}_{1}(\varphi)-D \mathcal{P}_{1}(\varphi)\right]
$$

Thus the last part of theorem 6.1 shows that the minimizers $\tilde{\varphi}$ of $\mathcal{J}$ are solutions of

$$
D \mathcal{F}_{1}(\varphi)=\beta^{-1} D \mathcal{P}_{1}(\varphi)
$$

This may be regarded as a linear eigenproblem with $\beta^{-1}$ as an eigenvalue. Substitute the expressions for these derivatives from proposition 5.1 and consider the eigenproblem of finding the values of $\mu$ such that there are non-trivial solutions $\varphi \in H_{0}(\Delta, \Omega)$ of

$$
\int_{\Omega}(\Delta \varphi \Delta \psi-\mu \rho \nabla \varphi \cdot \nabla \psi) d^{n} x+|\Omega| M(\Delta \varphi) M(\Delta \psi)=0
$$

for all $\psi \in H_{0}(\Delta, \Omega)$. This is the weak form of the system

$$
\begin{aligned}
-\Delta^{2} \varphi & =\mu \operatorname{div}(\rho \nabla \varphi) \quad \text { on } \Omega \text { subject to, } \\
\Delta \varphi+I(\Delta \varphi) & =0 \quad \text { and } \quad \varphi=0 \text { on } \partial \Omega .
\end{aligned}
$$

Here $I(\chi):=\int_{\Omega} \chi d^{n} x$ is the integral of $\chi$ over $\Omega$.

The following theorem proves that the least eigenvalue of this biharmonic eigenproblem (6.4) yields the optimal constant in the inequalities (4.10) and (1.2).

Theorem 6.2. Assume A1, A2 hold, $C_{2}$ is the optimal constant in (4.10) and $\beta$ as above. Then the least eigenvalue $\mu_{1}$ of the system (6.4) obeys $\mu_{1}=C_{2}=\beta^{-1}$ and equality holds in (4.10) for any eigenfunction corresponding to the eigenvalue $\mu_{1}$.

Proof. When $\varphi_{1}$ is a maximizer of $\mathcal{P}_{1}$ on C, then it will be a solution of (6.3) using linearity and theorem 6.1. Hence it is a solution of (6.4) with $\mu=\beta^{-1}$. Let $(\mu, \tilde{\varphi})$ be any eigenvalue and a corresponding eigenfunction of (6.4). Put $\psi=\varphi=\tilde{\varphi}$ in (6.4), then $\mathcal{F}_{1}(\tilde{\varphi})=\mu \mathcal{P}_{1}(\tilde{\varphi})$. Use (5.6), then

$$
0=\mathcal{F}_{1}(\tilde{\varphi})-\mu \mathcal{P}_{1}(\tilde{\varphi}) \geq(1-\mu \beta) \mathcal{F}_{1}(\tilde{\varphi}) .
$$

This implies $\mu \geq \beta^{-1}$ or $\beta^{-1}$ is the least eigenvalue of (6.4). 
7. THE CASE $\rho \equiv 1$.

When $\rho \equiv 1$ on $\Omega$, this biharmonic eigenproblem can be reduced to an eigenproblem for the Laplacian with zero Dirichlet data.

Given $\varphi \in H_{0}(\Delta, \Omega)$, let $\psi=L \varphi:=\Delta \varphi+I(\Delta \varphi)$. If $\varphi$ is an eigenfunction of the system (6.5)-(6.6) with $\rho \equiv 1$, then, from the boundary conditions, the corresponding $\psi$ will be in $H_{0}^{1}(\Omega)$ and will be a solution of

$$
-\Delta \psi=\mu I_{1} \psi \quad \text { on } \Omega \text {. }
$$

Here $I_{1}$ is the linear mapping of $L^{2}(\Omega)$ to itself defined by

$$
I_{1} \psi:=\psi-(1+|\Omega|)^{-1} I \psi
$$

Observe that $I_{1}$ is a rank 1 perturbation of the identity on $L^{2}(\Omega)$ and it is continuous and self-adjoint. Take inner products of this with $\psi$ and use Schwarz inequality to see that

$$
\frac{1}{1+|\Omega|}\|\psi\|^{2} \leq\left\langle I_{1} \psi, \psi\right\rangle \leq\|\psi\|^{2} \quad \text { for all } \psi \in L^{2}(\Omega) .
$$

In particular $I_{1}$ is a positive definite, self-adjoint, operator on $L^{2}(\Omega)$.

This inequality leads to the following bounds on the best constant $C_{2}$ in terms of the first eigenvalue $\lambda_{1 D}$ of the Dirichlet Laplacian on $\Omega$.

Theorem 7.1. Assume (A1), $\rho \equiv 1$ and $\lambda_{1 D}$ is the least eigenvalue of the Dirichlet Laplacian on $\Omega$. Then the optimal constant in (4.10) obeys

$$
\lambda_{1 D} \leq C_{2} \leq(1+|\Omega|) \lambda_{1 D}
$$

Proof. From Rayleigh's principle the least eigenvalue $\mu_{1}$ of (7.1) is given by the minimum value of

$$
R(\psi):=\frac{\|\nabla \psi\|^{2}}{\left\langle I_{1} \psi, \psi\right\rangle}
$$

for $\psi \in H_{0}^{1}(\Omega) \backslash\{0\}$. The inequality (7.3) then yields (7.4).

\section{A 2D CURL COERCIVITy INEQUALITy}

When $\Omega$ is a planar region, the inequality (4.10) enables us to prove (1.3) for all fields in a subspace of $H(\operatorname{curl}, \Omega)$.

First the relevant definitions. When $\psi \in H^{1}(\Omega)$, then $\operatorname{Curl} \psi$ is the vector field whose Cartesian components are $\left(D_{2} \psi,-D_{1} \psi\right)$. If $v \in L^{2}\left(\Omega ; \mathbb{R}^{2}\right)$, then its (weak) curl will be the distribution $W_{v}$ defined by

$$
W_{v}(\varphi):=\int_{\Omega} v \cdot \operatorname{Curl} \varphi d^{2} x \quad \text { for all } \varphi \in C_{c}^{\infty}(\Omega) .
$$

When $v(x):=\operatorname{Curl} \psi(x)$ then $|v(x)|=|\nabla \psi(x)|$ and $\operatorname{curl} v(x)=-\Delta \psi(x)$. 
If there is a function $\omega \in L_{l o c}^{1}(\Omega)$ such that

$$
W_{v}(\varphi)=-\int_{\Omega} \omega \varphi d^{2} x \quad \text { for all } \varphi \in C_{c}^{\infty}(\Omega),
$$

then we say that $\omega=\operatorname{curl} v$ on $\Omega$. When $v$ is differentiable, the Gauss-Green theorem yields the classical definition

$$
\operatorname{curl} v(x):=D_{1} v_{2}(x)-D_{2} v_{1}(x)
$$

Define $H(\operatorname{curl}, \Omega)$ to be the subspace of all fields in $L^{2}\left(\Omega ; \mathbb{R}^{2}\right)$ having $\operatorname{curl} v$ in $L^{2}(\Omega)$. $H(\operatorname{curl}, \Omega)$ is a real Hilbert space under the inner product

$$
[v, w]_{\operatorname{curl}}:=\int_{\Omega}[v(x) \cdot w(x)+\operatorname{curl} v(x) \operatorname{curl} w(x)] d^{2} x .
$$

The associated norm will be denoted $\|\cdot\|_{\text {curl }}$.

A field $v \in L^{2}\left(\Omega ; \mathbb{R}^{2}\right)$ is said to be irrotational whenever

$$
\int_{\Omega} v \cdot \operatorname{Curl} \varphi d^{2} x=0 \quad \text { for all } \varphi \in C_{c}^{\infty}(\Omega) .
$$

The class of all irrotational vector fields in $H(\operatorname{curl}, \Omega)$ will be denoted $N(\operatorname{curl})$ and is a closed subspace of $L^{2}\left(\Omega ; \mathbb{R}^{2}\right)$ and of $H(\operatorname{curl}, \Omega)$.

Suppose $v, \operatorname{curl} v$ are in $L^{1}\left(\Omega ; \mathbb{R}^{2}\right), L^{1}(\Omega)$ respectively, and $\tau:=\left(\nu_{2},-\nu_{1}\right)$ is a unit tangent field on $\partial \Omega$. Then (2.8) and (A1) imply that

$$
\int_{\Omega} \operatorname{curl} v d^{2} x=\int_{\partial \Omega} v \cdot \tau d s
$$

In consequence, (1.3) cannot hold for any irrotational field. Note that, from Schwarz inequality, for all $v \in H(\operatorname{curl}, \Omega)$,

$$
\left(\int_{\partial \Omega} v \cdot \tau d s\right)^{2} \leq|\partial \Omega| \int_{\partial \Omega}|v \cdot \tau|^{2} d s
$$

When $v \in H(\operatorname{curl}, \Omega)$, the left hand side of this inequality is always finite - but the right hand side need not be. Thus (1.3) is significantly stronger than the analogous (3d) result described in corollary 3.51 of [8].

We shall show that (1.3) holds on the orthogonal complement of $N$ (curl) in $H(\operatorname{curl}, \Omega)$. Define $\operatorname{Curl}_{0}^{1}(\Omega):=\left\{\operatorname{Curl} \psi: \psi \in H_{0}(\Delta, \Omega)\right\}$. Then $\operatorname{Curl}_{0}^{1}(\Omega)$ is a subspace of $H(\operatorname{curl}, \Omega)$ and the following analog of theorem 4.1 holds.

Theorem 8.1. Assume $\Omega \subset \mathbb{R}^{2}$ satisfies (A1). Then $\operatorname{Curl}_{0}^{1}(\Omega)$ is the orthogonal complement of $N(\operatorname{curl})$ in $H(\operatorname{curl}, \Omega)$.

Proof. When $v \in H(\operatorname{curl}, \Omega)$ and $\psi \in H_{0}^{1}(\Omega)$, use the Gauss-Green theorem to see that

$$
\int_{\Omega} v \cdot \operatorname{Curl} \psi d^{2} x=\int_{\Omega} \psi \operatorname{curl} v d^{2} x .
$$


Thus the subspaces $\operatorname{Curl}_{0}^{1}(\Omega)$ and $N(\operatorname{curl})$ are $L^{2}$-orthogonal and also orthogonal in $H(\operatorname{curl}, \Omega)$.

Substitute $H(\operatorname{curl}, \Omega)$, curl $u$ for $H(\operatorname{div}, \Omega)$, div $u$ in the definition (4.2) of a Lagrangian and repeat the proof with the obvious changes. Given $v \in H(\operatorname{curl}, \Omega)$, we find a $\hat{u} \in N(\operatorname{curl})$ and a $\hat{\psi} \in H_{0}(\Delta, \Omega)$ such that $v=\hat{u}+\operatorname{Curl} \hat{\psi}$. This is the desired orthogonal decomposition and $\operatorname{Curl}_{0}^{1}(\Omega)$ will be a closed subspace of $H(\operatorname{curl}, \Omega)$ just as was the case for $G_{0}^{1}(\Omega)$.

This result enables the proof that (1.3) holds on the subspace $\operatorname{Curl}_{0}^{1}(\Omega)$.

Theorem 8.2. Assume (A2) holds and $\Omega \subset \mathbb{R}^{2}$ satisfies (A1). Then (1.3) holds for all $v \in H(\operatorname{curl}, \Omega)$ which are $L^{2}$ - orthogonal to $N(\operatorname{curl})$. Moreover $C_{2}=\mu_{1}$ is the least eigenvalue of (6.4).

Proof. Substitute $v=\operatorname{Curl} \psi$ in (1.3). Then

$$
\int_{\Omega}|\Delta \psi|^{2} d^{2} x+|\Omega|^{-1}\left(\int_{\partial \Omega}(\nabla \psi) \cdot \nu d s\right)^{2} \geq C_{2} \int_{\Omega} \rho|\nabla \psi|^{2} d^{2} x .
$$

upon using the relationship between $\tau$ and $\nu$ on $\partial \Omega$. From theorem 6.2, this holds for all $\psi \in H_{0}(\Delta, \Omega)$ with $C_{2}=\mu_{1}$. Hence (1.3) holds for all $v \in \operatorname{Curl}_{0}^{1}(\Omega)$. The preceding theorem 8.1 now yields this theorem.

\section{REFERENCES}

[1] G. Auchmuty, "Duality for Non-Convex Variational Principles", J. Differential Equations, 50 (1983) 80-145.

[2] G. Auchmuty, "Orthogonal Decompositions and Bases for Three-dimensional Vector Fields", Numerical Functional Analysis and Optimization, 15 (1994) 455-488.

[3] G. Auchmuty, "Optimal Coercivity Inequalities in $W^{1, p}(\Omega)$ ", (submitted).

[4] L.C. Evans, Partial Differential Equations, American Math. Society, (1998).

[5] L. C. Evans and R. F. Gariepy, Measure Theory and Fine Properties of Functions, CRC Press, Boca Raton (1992).

[6] G.P. Galdi, An Introduction to the Mathematical Theory of the Navier-Stokes Equations, Vol I, Springer Verlag, (1994).

[7] G. Hellwig, Differential Operators of Mathematical Physics Addison Wesley, Reading Mass., (1967).

[8] P. Monk, Finite Element Methods for Maxwell's Equations, Clarendon Press, Oxford (2003).

[9] J. Necas, Les Méthodes Directes en Théorie des Équations Elliptiques, Masson et Cie, Paris, (1967). USA

Department of Mathematics, University of Houston, Houston, TX 77204-3008,

E-mail address: auchmuty@uh.edu 